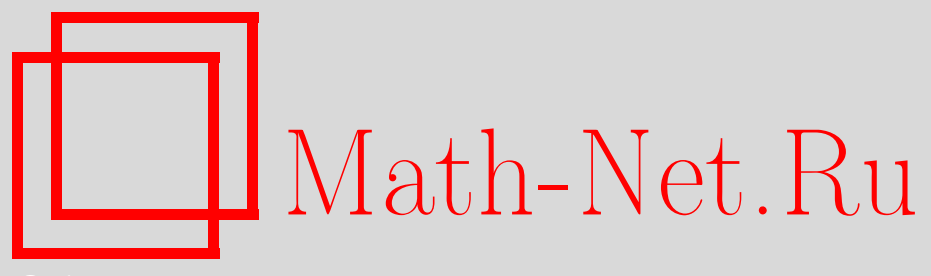

K. С. Рютин, О равномерно непрерывных операторах почти наилучшего обобщенного рационального приближения, Матем. заметки, 2010, том 87, выпуск 1, 147150

DOI: https://doi.org/10.4213/mzm8554

Использование Общероссийского математического портала Math-Net.Ru подразумевает, что вы прочитали и согласны с пользовательским соглашением http://www.mathnet.ru/rus/agreement

Параметры загрузки:

IP: 54.162 .127 .20

26 апреля 2023 г., 14:24:30

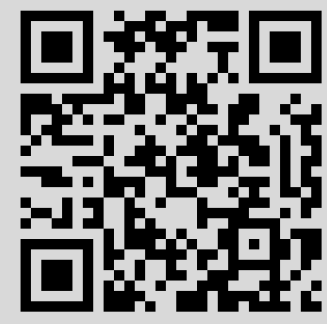




\section{О равномерно непрерывных операторах почти наилучшего обобщенного рационального приближения}

\section{К. С. Рютин}

Пусть $X$ - нормированное пространство, $M \subset X, \varepsilon \geqslant 0$. Отображение $\Phi: X \rightarrow M$ называется мультипликативной $\varepsilon$-выборкой из $X$ на $M$, если для всех $x \in X$ выполнено

$$
\|\Phi(x)-x\| \leqslant(1+\varepsilon) \rho(x, M), \quad \text { где } \quad \rho(x, M)=\inf _{m \in M}\|x-m\| .
$$

Выборка называется непрерывной (равномерно непрерывной), если $\Phi$ непрерывно (равномерно непрерывно). Отметим, что при $\varepsilon=0$ получается определение выборки из метрической проекции.

Пусть $K$ - связный метрический компакт, $X=C(K)$ и задана пара подпространств $(V, W)$ в $X$ такая, что $\operatorname{dim} V=m, \operatorname{dim} W=n$, где $m, n \in \mathbb{N}$. Всюду ниже

$$
W^{+}=\{w \in W: w(\tau) \geqslant 0 \text { для любого } \tau \in[0 ; 1]\},
$$

$B$ - единичный шар в $X$, a $\|\cdot\|$ означает норму в $X$. Предположим, что существует функция $w_{0} \in W$ такая, что $w_{0}>0$ на $K$ и что $A(w)=\{\tau \in K: w(\tau) \neq 0\}$ - открытое всюду плотное подмножество компакта $K$ для произвольной функции $w \in W \backslash\{0\}$. Рассмотрим множество обобщенных дробей

$$
\mathscr{R}=\mathscr{R}_{V, W}=\mathrm{Cl}\left\{r=\frac{v}{w}: v \in V, w \in W, w>0 \text { на } K\right\},
$$

где $\mathrm{Cl}$ означает замыкание в $X$. Алгебраические рациональные функции $\mathscr{R}_{m, n}$ и конечномерные подпространства являются примерами множеств обобщенных дробей.

Известно [1], [2], что оператор метрической проекции на множество $\mathscr{R}_{m, n}, n \geqslant 1$, в $C[0 ; 1]$ не является непрерывным, а оператор метрической проекции на множество алгебраических полиномов $\mathscr{P}_{n}, n \geqslant 1$, в $C[0 ; 1]$ не является равномерно непрерывным на шаре пространства. Из результатов [3] следует, что для любого $\varepsilon>0$ существует непрерывная $\varepsilon$-выборка из $C(K)$ на множество $\mathscr{R}_{V, W}$, но при некоторых условиях на $V, W$ (например, в случае $\mathscr{R}_{0, n}, n \geqslant 1$ ) при $\varepsilon<2$ не может быть равномерно непрерывной на шаре мультипликативной $\varepsilon$-выборки на $\mathscr{R}_{V, W}$ (см. [5]).

Теорема 1. Пусть $\mathscr{R}_{V, W} \cap B$ компактно. Тогда для любого $\varepsilon>0$ существует равномерно непрерывная мультипликативная $\varepsilon$-выборка $\Phi: B \rightarrow \mathscr{R}_{V, W}$.

Заметим, что множество $W^{+}$является непустым замкнутым выпуклым конусом в $W$ и $W^{+} \neq W$. Для любой точки $w \in \partial W^{+}$найдется $\tau \in K: w(\tau)=0$, а для любой точки $w \in \operatorname{int} W^{+}$справедливо неравенство $w(\tau)>0$ на $K$.

ЗАмечАниЕ 1. Множество $\mathscr{R}$ можно определить и другим (равносильным) способом, а именно $\mathscr{R}=\left\{v / w: v \in V, w \in W^{+} \backslash\{0\}\right\}$. Тем самым, для любых $v \in V, w \in W^{+} \backslash\{0\}$ дробь $r=v / w$ корректно определена, т.е. имеется функция $r \in X$ такая, что $r(\tau) \equiv$ $v(\tau) / w(\tau)$ на $A(w)$. Тем самым, можно доопределить отношение функций $v(\tau), w(\tau)$ на всем $K$ следующим образом: $v(\tau) / w(\tau)=r(\tau)$. В дальнейшем отношение $v / w$ мы будем понимать именно в таком смысле.

Работа выполнена при поддержке Российского фонда фундаментальных исследований (грант № 08-01-00208) и программы "Ведущие научные школы" (грант № НШ-3233.2008.1).

(C) К. С. Рютин, 2010 
В пространстве $W$ можно выбрать аффинную гиперплоскость $Г$ такую, что она будет пересекать каждый луч с началом в нуле, лежащий в $W^{+}$, ровно в одной точке и при этом для любой точки $w \in \Gamma \cap W^{+}$выполнено неравенство $\|w\| \geqslant 1$. Докажем это. Можно выбрать точки $\tau_{j} \in K, 1 \leqslant j \leqslant n$, и базис $\left\{\omega_{j}\right\}, 1 \leqslant j \leqslant n$, в $W$ такие, что $\omega_{i}\left(\tau_{j}\right)=\delta_{i j}, 1 \leqslant i, j \leqslant n$, где $\delta_{i j}$ - символ Кронекера. Пусть $w=\sum_{j=1}^{n} c_{j} \omega_{j} \in W^{+}$, где $c_{j} \in \mathbb{R}$; тогда $w\left(\tau_{j}\right) \geqslant 0$ для любого индекса $1 \leqslant j \leqslant n$. Следовательно, $c_{j} \geqslant 0$ для любого $1 \leqslant j \leqslant n$. В качестве $\Gamma$ можно взять следующую аффинную гиперплоскость: $\left\{\sum_{j=1}^{n} c_{j} \omega_{j}: \sum_{j=1}^{n} c_{j}=H\right\}$, где $H>0$ достаточно велико. Положим

$$
Q=\Gamma \cap W^{+}, \quad \Lambda=\{(v, w): v \in V, w \in Q\}
$$

и определим отображение $T: \Lambda \rightarrow \mathscr{R}$ следующим образом: $T(v, w)=v / w$. Отметим, что множество $Q$ выпуклое и компактное. Следовательно, существует число $a_{0}>0$ такое, что $\|w\| \leqslant a_{0}$ при $w \in Q$.

ЗАмечАниЕ 2. Для любого числа $\alpha>0$ найдется число $q=q(\alpha)>0$ такое, что для всех $w_{1}, w \in Q$ найдется точка $w_{0} \in Q$ такая, что $\left\|w-w_{0}\right\| \leqslant \alpha$ и на $K$ выполнено неравенство $w_{1}(\tau) \leqslant q w_{0}(\tau)$.

Рассмотрим функционал $g_{\alpha}(w)=\max _{z \in Q}:\|z-w\| \leqslant \alpha \min _{\tau \in K} z(\tau)$. Заметим, что для любого $w \in Q$ выполнено неравенство $g_{\alpha}(w)>0$. В самом деле, найдется функция $\widehat{w} \in Q$, $\widehat{w} \neq w$, такая, что $\widehat{w}>0$ на $K$. Взяв $z$ из отрезка $[w ; \widehat{w}]$, получим неравенство $g_{\alpha}(w)>0$. Из компактности $Q$ следует, что найдется $q_{1}(\alpha)=\min _{w \in Q} g_{\alpha}(w)>0$. Положив $q(\alpha)=$ $a_{0} / q_{1}(\alpha)$, установим справедливость замечания.

Проверим непрерывность отображения $T$. Пусть (от противного) имеется последовательность $y_{j}=\left(v_{j}, w_{j}\right) \rightarrow y_{0}=\left(v_{0}, w_{0}\right), j \in \mathbb{N}$, и при этом для всякого $j \in \mathbb{N}$ справедливо неравенство $\left\|T\left(y_{j}\right)-T\left(y_{0}\right)\right\| \geqslant h>0$ (разумеется, $y_{j} \in \Lambda, j \in \mathbb{Z}^{+}$). Возможны два случая:

1) последовательность $\left\|T\left(y_{j}\right)\right\|$ ограничена и, следовательно, можно выделить подпоследовательность $y_{j} \rightarrow y_{0}$ такую, что $T\left(y_{j}\right) \rightarrow r \in \mathscr{R}$;

2) можно выделить подпоследовательность $y_{j}$ такую, что $\left\|T\left(y_{j}\right)\right\| \rightarrow \infty$.

Покажем, что случай 2) невозможен. Можно подобрать последовательность чисел $c_{j}>0$, $c_{j} \rightarrow 0$ при $j \rightarrow \infty$, для которой при любом $j \in \mathbb{N}$ выполнено $\left\|c_{j} T\left(y_{j}\right)\right\|=1$. Считаем, что $c_{j} T\left(y_{j}\right) \rightarrow \widetilde{r} \in \mathscr{R}$ при $j \rightarrow \infty$. Заметим, что $\widetilde{r} \equiv 0$ на множестве $A\left(w_{0}\right)$ и, следовательно, $\widetilde{r} \equiv 0$. Последнее противоречит тому, что $\|\widetilde{r}\|=1$. Аналогичным образом в 1-ом случае можно показать, что $r \equiv v_{0} / w_{0}$.

Пусть $D=\{y \in \Lambda:\|T(y)\| \leqslant 10\}$. Заметим, что $D$ - компактное подмножество $\Lambda$. В самом деле, ограниченность $D$ очевидна, а замкнутость легко следует из компактности $Q$ и замечания 1. Всюду далее $\rho(x)=\rho(x, \mathscr{R}), \rho_{j}=\rho\left(x_{j}, \mathscr{R}\right), j=1,2$.

Лемма 1. Пусть $\varepsilon \in(0 ; 1), a$

$$
Z(x):=\{z \in \Lambda:\|T(z)-x\| \leqslant(1+\varepsilon) \rho(x)\},
$$

где $x \in B$. Тогда $Z(x)$ - ограниченное выпуклое замкнутое подмножество $\Lambda$.

ДоказАтельство. Заметим, что для любого $x \in B$ справедливо включение $Z(x) \subset D$. Отсюда следует, что $Z(x)$ - компактное подмножество $\Lambda$. Проверим выпуклость $Z(x)$.

Следующая конструкция была фактически использована Конягиным в работе [3]. Пусть $\left(v_{1}, w_{1}\right),\left(v_{2}, w_{2}\right) \in Z(x)$; докажем, что точка $\left(v_{\lambda}, w_{\lambda}\right) \in Z(x)$, где

$$
v_{\lambda}=\lambda v_{1}+(1-\lambda) v_{2}, \quad w_{\lambda}=\lambda w_{1}+(1-\lambda) w_{2}, \quad \text { для любого } \quad \lambda \in[0 ; 1] .
$$

Для любой точки $\tau \in A\left(w_{1}\right) \cap A\left(w_{2}\right)$ имеем $\left|v_{j}(\tau)-x(\tau) w_{j}(\tau)\right| \leqslant(1+\varepsilon) \rho(x) w_{j}(\tau), j=1,2$. Поэтому

$$
\begin{aligned}
\left|v_{\lambda}(\tau)-x(\tau) w_{\lambda}(\tau)\right| & \leqslant \lambda\left|v_{1}(\tau)-x(\tau) w_{1}(\tau)\right|+(1-\lambda)\left|v_{2}(\tau)-x(\tau) w_{2}(\tau)\right| \\
& \leqslant \lambda(1+\varepsilon) \rho(x) w_{1}(\tau)+(1-\lambda)(1+\varepsilon) \rho(x) w_{2}(\tau)=(1+\varepsilon) \rho(x) w_{\lambda}(\tau)
\end{aligned}
$$


для любой точки $\tau \in A\left(w_{1}\right) \cap A\left(w_{2}\right)$. Тем самым, оценка $\left|T\left(v_{\lambda}, w_{\lambda}\right)-x\right| \leqslant(1+\varepsilon) \rho(x)$ выполнена на всюду плотном подмножестве $A\left(w_{1}\right) \cap A\left(w_{2}\right) \subset K$, а значит, и на всем $K$. Лемма доказана.

В $V \times W$ имеется норма $|(v, w)|:=\max \{\|v\|,\|w\|\}$, индуцирующая метрику на $V \times \Gamma$. Пусть $\mathscr{V}=\mathscr{V}(V \times \Gamma)$ - множество всех замкнутых ограниченных выпуклых подмножеств в $V \times \Gamma$. Заметим, что $\mathscr{V}$ - полное метрическое пространство относительно хаусдорфовой метрики

$$
h\left(A_{1}, A_{2}\right)=\max \left\{\sup _{a \in A_{1}} \inf _{b \in A_{2}}|a-b|, \sup _{b \in A_{2}} \inf _{a \in A_{1}}|a-b|\right\},
$$

где $A_{1}, A_{2} \in \mathscr{V}$. Из результатов [4] следует, что определено отображение $S t: \mathscr{V} \rightarrow V \times \Gamma$ (множеству $A$ сопоставляется его точка Штейнера) такое, что при некотором $C>0$ для любых $A, B \in \mathscr{V}$ выполнены условия:

1) $\operatorname{St}(A) \in A$,

2) $|S t(A)-S t(B)| \leqslant C h(A, B)$.

ДокАЗАТЕЛЬСтво теоремы. 1) Зафиксируем произвольное число $\varepsilon \in(0 ; 1)$. Требуемую в теореме выборку зададим так: $\Phi:=T \circ S t \circ Z$; многозначное отображение $Z: B \rightarrow \mathscr{V}(V \times \Gamma)$ определено в лемме 1 . Необходимо проверить, что отображение $\Phi$ будет равномерно непрерывным на шаре $B$. Заметим, что для любого $x \in B$ справедливо включение $Z(x) \subset D$, а ограничение $T$ на $D$ является равномерно непрерывным.

Основная сложность - проверка равномерной непрерывности $Z$. Для $x \in B, \nu \geqslant 0$ положим

$$
Z(x, \nu):=\{z \in \Lambda:\|T(z)-x\| \leqslant(1+\varepsilon) \rho(x)+\nu\} .
$$

Заметим, что $Z(x, 0)=Z(x)$. Нам понадобится следующая

Лемма 2. Для любых $\xi, b>0$ найдется число $\nu>0$ такое, что для любой точки $x \in B: \rho(x) \geqslant b$, выполнено неравенство $h(Z(x), Z(x, \nu))<\xi$.

ДоказАтельство. Зафиксируем $b>0$ и $\xi \in(0 ; d), d=\operatorname{diam} D$. Пусть $y_{1}=\left(v_{1}, w_{1}\right) \in$ $Z(x, \nu)$. Покажем, что если $\nu>0$ достаточно мало, то найдется точка $y_{2} \in Z(x)$ такая, что $\left|y_{1}-y_{2}\right| \leqslant \xi$. Заметим, что существует точка $y_{0} \in Z(x)$, для которой $\left\|T\left(y_{0}\right)-x\right\| \leqslant$ $(1+\varepsilon / 2) \rho(x)$, и для любого $\tau \in K$ выполнено неравенство

$$
w_{1}(\tau) \leqslant q w_{0}(\tau), \quad \text { где } \quad q=q(\varepsilon, b)>0 .
$$

В самом деле, найдется точка $\widetilde{y} \in Z(x):\|T(\widetilde{y})-x\| \leqslant(1+\varepsilon / 10) \rho(x)$. Из равномерной непрерывности отображения $T$ следует, что при $|z-\widetilde{y}| \leqslant \omega(b), z \in \Lambda$, выполнено неравенство $\|T(z)-T(\widetilde{y})\| \leqslant b \varepsilon / 10 \leqslant \varepsilon \rho(x) / 10$, где $\omega(b)>0$. Воспользовавшись замечанием 2 при $\alpha=\omega(b)$, построим требуемую точку $y_{0}$. Покажем, что в качестве точки $y_{2}$ можно взять $y_{2}=(1-\lambda) y_{0}+\lambda y_{1}$, где $\lambda=1-\xi / 2 d \in(1 / 2 ; 1]$.

Во-первых,

$$
\left|y_{1}-y_{2}\right|=(1-\lambda)\left|y_{1}-y_{0}\right| \leqslant(1-\lambda) \operatorname{diam} Z(x, \nu) .
$$

Но при $\nu<1$ выполнено включение $Z(x, \nu) \subset D$. Следовательно, справедливо неравенство $\left|y_{1}-y_{2}\right| \leqslant(1-\lambda) d=\xi / 2$.

Во-вторых, покажем, что $y_{2} \in Z(x)$. Для любой точки $\tau \in K$ выполнено неравенство

$$
\begin{aligned}
\left|x(\tau) w_{2}(\tau)-v_{2}(\tau)\right| & \leqslant(1-\lambda)\left|x(\tau) w_{0}(\tau)-v_{0}(\tau)\right|+\lambda\left|x(\tau) w_{1}(\tau)-v_{1}(\tau)\right| \\
& \leqslant(1-\lambda)\left(1+\frac{\varepsilon}{2}\right) \rho(x) w_{0}(\tau)+\lambda[(1+\varepsilon) \rho(x)+\nu] w_{1}(\tau) \\
& =(1+\varepsilon) \rho(x)\left[(1-\lambda) w_{0}(\tau)+\lambda w_{1}(\tau)\right]-\frac{\varepsilon}{2}(1-\lambda) \rho(x) w_{0}(\tau)+\lambda \nu w_{1}(\tau) .
\end{aligned}
$$


Из нашего выбора $w_{0}$ следует, что

$$
\begin{aligned}
-\frac{\varepsilon}{2}(1-\lambda) \rho(x) w_{0}(\tau)+\lambda \nu w_{1}(\tau) & \leqslant-\frac{\varepsilon}{2}(1-\lambda) \rho(x) w_{0}(\tau)+\lambda \nu q w_{0}(\tau) \\
& \leqslant w_{0}(\tau)\left[-\frac{\varepsilon \xi b}{4 d}+\left(1-\frac{\xi}{2 d}\right) q(\varepsilon, b) \nu\right] \leqslant 0
\end{aligned}
$$

последнее неравенство справедливо при достаточно малом $\nu>0$. Итак, при малом $\nu>0$ в произвольной точке $\tau \in K$ выполнено $\left|x(\tau) w_{2}(\tau)-v_{2}(\tau)\right| \leqslant(1+\varepsilon) \rho(x) w_{2}(\tau)$. Тем самым, $y_{2} \in Z(x)$. Лемма доказана.

Надо показать, что для любого числа $a>0$ существует постоянная $\delta>0$ такая, что для любых точек $x_{1}, x_{2} \in B: \mid x_{1}-x_{2} \| \leqslant \delta$ выполнено неравенство $\left\|\Phi\left(x_{1}\right)-\Phi\left(x_{2}\right)\right\| \leqslant a$. Зафиксируем малое число $a>0$. Пусть $x_{1}, x_{2} \in B ; y_{1} \in Z\left(x_{1}\right), y_{2} \in Z\left(x_{2}\right)$; тогда

$$
\left\|T\left(y_{1}\right)-T\left(y_{2}\right)\right\| \leqslant(1+\varepsilon)\left(\rho_{1}+\rho_{2}\right)+\left\|x_{1}-x_{2}\right\| .
$$

Следовательно, можно рассматривать только такие точки $x_{1}, x_{2} \in B$, что $\rho_{1}+\rho_{2} \geqslant$ $k\left\|x_{1}-x_{2}\right\|$, где $k$ - большая константа. Кроме того, всегда выполнено $\left|\rho_{1}-\rho_{2}\right| \leqslant\left\|x_{1}-x_{2}\right\|$. Из наших рассмотрений следует, что достаточно разобрать случай $\rho\left(x_{j}\right) \geqslant a / 10$ при $j=1,2$.

Надо показать, что для любого $\xi>0$ найдется $\delta>0$ такое, что для любых $x_{1}, x_{2} \in B$ таких, что $\left\|x_{1}-x_{2}\right\| \leqslant \delta$ и $\rho\left(x_{j}\right) \geqslant a / 10, j=1,2$, выполнено неравенство $h\left(Z\left(x_{1}\right), Z\left(x_{2}\right)\right) \leqslant \xi$. Пусть $y_{2} \in Z\left(x_{2}\right)$; тогда

$$
\left\|T\left(y_{2}\right)-x_{1}\right\| \leqslant\left\|x_{1}-x_{2}\right\|+\left\|T\left(y_{2}\right)-x_{2}\right\| \leqslant \delta(2+\varepsilon)+(1+\varepsilon) \rho\left(x_{1}\right) .
$$

Следовательно, $y_{2} \in Z\left(x_{1}, \delta(2+\varepsilon)\right)$. Применив лемму 2 при $b=a / 10$, получим (при достаточно малом $\delta$ ) точку $y_{1} \in Z\left(x_{1}\right)$, для которой $\left|y_{1}-y_{2}\right| \leqslant \xi$. Для завершения доказательства достаточно воспользоваться равномерной непрерывностью $T$ и липшицевостью $S t$. Теорема доказана.

Имеется целая серия примеров множеств $\mathscr{R}_{V, W}, \mathrm{k}$ которым применима теорема 1. Точнее, для любых чисел $m, n \in \mathbb{N}$ найдется пара подпространств $V, W \subset C[0 ; 1]$ таких, что $\operatorname{dim} V=m, \operatorname{dim} W=n$ и множество $\mathscr{R}_{V, W} \cap B$ непусто и компактно, где $B-$ единичный шар в $C[0 ; 1]$.

Рассмотрим полиномы $e_{j}(\tau)=\prod_{k \neq j}\left(\tau-\tau_{k}\right)^{2}$, точки $\tau_{k}=k / n \in[0 ; 1], 1 \leqslant j, k \leqslant n$, и положим $W=\left\langle\left\{e_{j}\right\}_{1 \leqslant j \leqslant n}\right\rangle$. Если $f(\tau)=\sum_{j=1}^{n} c_{j} e_{j}(\tau) \geqslant 0$ на $[0 ; 1]$, то $f\left(\tau_{j}\right) \geqslant 0$ для любого $1 \leqslant j \leqslant n$ и, следовательно, $c_{j} \geqslant 0$ для любого $1 \leqslant j \leqslant n$. Заметим, что функция $f \in W^{+} \backslash\{0\}$ может обращаться в нуль только в точках из множества $\left\{\tau_{j}\right\}$. Более того, $f\left(\tau_{j}\right)=0$ тогда и только тогда, когда $c_{j}=0$. Подберем $V$ (например, состоящим из полиномов) таким образом, чтобы у любой функции $v \in V$ порядок нуль в точках $\tau_{j}$ был не меньше 3. Тогда для любых $v \in V, w \in W^{+} \backslash\{0\}$ будет функция $v(\tau) / w(\tau)$ определена на всем отрезке $[0 ; 1]$ и множество $\mathscr{R}_{V, W} \cap B$ будет компактно.

Автор благодарит С. В. Конягина за многочисленные полезные обсуждения и поддержку.

\section{СПИСОК ЦИТИРОВАННОЙ ЛИТЕРАТУРЫ}

[1] H. Maehly, Ch. Witzgall, Numer. Math., 2:5 (1960), 293-307. [2] H. Werner, Math. Z., 86:4 (1964), 317-326. [3] С. В. Конягин, Матем. заметки, 44:3 (1988), 404. [4] Е. Д. Посицельский, Оптимизация, 1971, № 4 (21), 83-89. [5] К. С. Рютин, Матем. заметки, 71:2 (2002), 261-270.

\section{К. С. Рютин}

Поступило

Московский государственный

16.01.2009

университет им. М. В. Ломоносова

E-mail: ksrutin@zmail.ru 